\title{
Quaderni
}

QUADERN I Communication, technologies, pouvoir

\section{Gouverner par la comparaison : usages et mésusages des comparaisons internationales des systèmes de santé}

\section{Sébastien Guigner}

\section{(2) OpenEdition \\ Journals}

Édition électronique

URL : http://journals.openedition.org/quaderni/739

DOI : $10.4000 /$ quaderni.739

ISSN : 2105-2956

Éditeur

Les éditions de la Maison des sciences de l'Homme

\section{Édition imprimée}

Date de publication : 5 octobre 2013

Pagination : $27-38$

Référence électronique

Sébastien Guigner, "Gouverner par la comparaison : usages et mésusages des comparaisons internationales des systèmes de santé », Quaderni [En ligne], 82 | Automne 2013, mis en ligne le 05 octobre 2015, consulté le 02 mai 2019. URL : http://journals.openedition.org/quaderni/739 ; DOI : 10.4000/quaderni.739 


\section{$D$ ossier}

\section{Gouverner par la comparaison :}

« L'intérêt et la nécessité des comparaisons internationales est indéniable », c'est en ces mots qu'au printemps 2012 le président du Haut Comité de Santé Publique présente le rapport sur l'état de santé et l'efficacité du système de santé

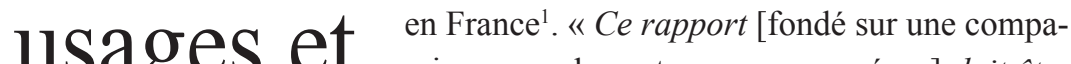
USAGCS C raison avec les autres pays européens] doit être mésusages des perçu comme une sorte de boussole indispensable pour ceux qui, en France, ont des responsabilités en matière de santé publique ", poursuit-il. La comparaisons internationales des systèmes de santé

\section{Sébastien Guigner}

Maître de conférences en science politique, Sciences Po Bordeaux, Centre Émile Durkheim croyance en la vertu des comparaisons internationales n'est pas propre au HCSP, ni au domaine de la santé, elle s'inscrit dans une dynamique plus large où l'action de gouverner se doit de plus en plus de se draper d'objectivité2. Peut-être plus que dans tout autre domaine toutefois, dans la santé la comparaison s'est érigée en totem de l'action publique. La frénésie comparative touche tous les organismes (HCCAM, IRDES, DREES, etc.) prétendant éclairer les choix des pouvoirs publics, tandis qu'en la matière les chercheurs académiques s'apparentent fréquemment à des «policy scientists » manipulant les outils de l'analyse des politiques publiques pour délivrer "des préconisations "prêtes à penser » aux décideurs $»^{3}$. Visiblement ces derniers valident la démarche, chacun a en effet pu constater la fréquence à laquelle les acteurs politiques invoquent l'étranger lorsqu'il s'agit de justifier leurs choix de politiques publiques. La comparaison internationale est ainsi devenue un élément clef de la légitimation des réformes des systèmes de santé, sinon l'argument ultime.

Passage obligé donc, la comparaison internationale n'est toutefois pas qu'un outil pratique d'aide à la décision, elle n'est pas une technique neutre qui permet simplement d'informer et d'opéra- 
tionnaliser plus ou moins directement l'action publique. La comparaison fonctionne en réalité comme un «instrument de gouvernement $»^{4}$. En peu de mots, elle est un dispositif qui structure fondamentalement l'action publique. D'une part, les comparaisons portent et confortent des représentations spécifiques de l'enjeu traité. D'autre part, elles influent sur les rapports de force. Sous couvert de dépolitisation et de rationalisation de la décision, la comparaison internationale est un outil politique stratégique; elle sert souvent plus à biaiser les choix politiques qu'à les informer.

\section{Un instrument d'apprentissage du change- ment}

Plusieurs objectifs peuvent sous-tendre les comparaisons internationales des systèmes de santé5. Dans leur version la moins ambitieuse les comparaisons peuvent simplement viser, par effet de contraste, une meilleure connaissance des dispositifs étrangers et nationaux. Les comparaisons peuvent également chercher à générer des explications causales des systèmes de santé et montrer que certains facteurs supposés clefs ne le sont pas tant que cela alors que d'autres qui sont négligés apparaissent décisifs. Mais au fond chacun de ces objectifs sert l'objectif ultime de la comparaison des systèmes de santé : apprendre des autres pour améliorer les dispositifs. Identifier et propager les bonnes pratiques - parfois à travers le benchmarking ${ }^{6}$ - est effectivement la motivation centrale des comparaisons des politiques et systèmes de santé menées par les divers organismes de conseil.

Le succès de la perspective comparée ne se limite pas au monde politique, la sphère académique a elle aussi succombé au chant des sirènes et pleinement contribué à faire de la comparaison internationale des politiques de santé une « industrie en expansion $»^{7}$. Bien qu'il soit généralement affirmé moins explicitement que par les institutions publiques, l'objectif d'apprentissage est partagé par bon nombre d'études comparatives menées dans le champ académique - qui lui doivent leur financement ${ }^{8}$. Principalement dans les disciplines prescriptives, telles que l'économie, le management et santé publique, mais aussi en sociologie et science politique. De fait, la comparaison y est couramment conçue comme un outil indispensable d'innovation et d'aide à la décision. Ainsi, A. Schneider et $\mathrm{H}$. Ingram d'affirmer que « à moins que l'analyse ne mettent en lumière l'exemple des autres pays, les changements seront marginaux $»{ }^{9}$. Pour R. Blank et V. Burau, auteurs d'un ouvrage de référence sur la comparaison des politiques de santé, « seules les études comparatives peuvent générer les données nécessaires pour examiner la gamme complète des options politiques ${ }^{10}$.

Potentiellement, tirer des leçons pour l'action publique des choix effectués ailleurs peut aussi bien mener à décrier la situation nationale qu'à la valoriser et ainsi conforter des dispositifs. Or, les comparaisons sont déployées parce que les systèmes de santé sont considérés comme problématiques - problèmes de financement, d'accessibilité, de performance, etc. Dès lors, ils appellent des solutions nouvelles. Dit autrement, dans la mesure où elles sont motivées par l'insatisfaction de l'existant ou la crainte du futur, il est peu probable que les comparaisons conduisent à conforter l'existant. Il est bien sûr des exceptions mais la « nature » des comparaisons internatio- 
nales à visée normative est d'apprendre à changer, la comparaison internationale est poussée par une envie de changement. C'est là un premier biais de ces comparaisons, leur résultat est surdéterminé.

\section{Des comparaisons souvent défaillantes}

Que les comparaisons internationales des systèmes de santé soient issues de la sphère académique ou non, elles contiennent souvent de nombreuses failles et pourraient à elles seules illustrer l'ensemble des critiques classiques adressées à l'exercice comparatif ${ }^{11}$. Le plus couramment ce sont, à juste titre, les limites de la comparaison des données qui sont soulignées (définition des termes, choix des critères, fiabilité des sources, etc.). L'OCDE s'autorise ainsi à publier des schémas comparatifs des rémunérations du personnel infirmier alors que pour certains pays les données ne concernent que les infirmières qualifiées, que d'autres pays livrent des données n'incluant pas les heures supplémentaires, que d'autres encore incluent les employées à temps partiel dans leurs données, que les tâches de ces personnels varient selon les pays, etc. ${ }^{12}$ Il ne s'agit là que d'un exemple parmi tant d'autres des limites des comparaisons de l'OCDE équivalentes sur ce point à celle de l'OMS - qui sont pourtant l'aliment de base de la plupart des analyses comparatives appliquées, des recherches comparatives académiques, ou des recherches effectuées par les medias grand public ${ }^{13}$.

Il convient de souligner d'autres failles tout aussi fondamentales et récurrentes mais plus rarement dénoncées. Ces comparaisons regorgent de mots valises tels que « qualité », « efficacité » ou « réformes » qui, bien qu'ils consti- tuent régulièrement l'objet des comparaisons, sont fort peu précisés. Par exemple, beaucoup de comparaisons internationales cherchent à expliquer et à évaluer les réformes de santé mais combien s'efforcent de définir et de questionner ce qu'elles prétendent observer ? Ainsi, l'observation de ces réformes est généralement statique et fait fi des démonstrations de la sociologie des organisations et de l'analyse des politiques publiques ${ }^{14}$. On postule que les décisions adoptées sont appliquées telles qu'elles, sans observer les déviations de la mise en œuvre, « on fait comme si ». Par ailleurs, sans définition, toute nouvelle disposition adoptée ici ou là est présentée comme une « réforme » alors que, par exemple, elle peut n'être que la consolidation de choix antérieurs ${ }^{15}$. Ces comparaisons relèvent ainsi fréquemment de « comparaisons ventriloques $»^{16}$ où l'observateur fait dire à la comparaison ce qu'il présuppose. Dans le cas précis, ce biais laisse à penser que le monde entier réforme et, par conséquent, qu'il est nécessaire de réformer ${ }^{17}$.

On peut également s'interroger sur l'intérêt d'effectuer des comparaisons inter-« nationales » des systèmes de santé. De fait, ces trente dernières années nombreux sont les pays à avoir vu les autorités infranationales accroître leur rôle dans le système de santé, y compris des pays comme la France ou le Royaume-Uni dont le système de santé est considéré comme extrêmement centralisé. Bien entendu des différences majeures persistent, selon les pays ces autorités sont plus ou moins locales et disposent de compétences fiscales, d'orientation stratégique ou de mise en œuvre, compétences qui peuvent en outre varier selon les domaines de santé. Au-delà de ces multiples combinaisons, il reste que les systèmes 
de santé sont aujourd'hui des dispositifs multiniveaux faisant interagir le national et l'infranational. De même, des institutions telles que l'UE jouent désormais un rôle décisif en matière de santé, pensons entre autres au contrôle des médicaments ou au temps de travail des personnels de santé. Enfin, les systèmes de santé ne sont pas isolés les uns des autres. Certains phénomènes affectant les systèmes de santé se jouent même à l'échelle du globe, le tourisme médical et la désertification médicale en sont des exemples. Au final, les systèmes de santé nationaux n'ont plus grand-chose de national. Territorialisation, transnationalisation et supranationalisation sont autant de dimensions que la comparaison des systèmes de santé devrait systématiquement intégrer ${ }^{18}$. En se tenant à l'écart de cette démarche, sur ce point comme sur celui de la réforme, l'instrument de la comparaison internationale opérationnalise des représentations sociales qu'il contribue ainsi à stabiliser ; l'espace politique de la santé serait l'espace national.

Non sans lien, l'absence de raisonnement systémique constitue une autre lacune majeure de ces comparaisons. Alors que l'expression « système » de santé est d'usage courant, on ne s'intéresse que rarement à ce qui fait système. Dans la plupart des comparaisons, les politiques et dispositifs du système sont isolés non seulement les uns des autres mais aussi de leur environnement juridique (par ex. responsabilité des professions médicales), politique (par ex. organisation des groupes de pression) ou sociétal (par ex. demandes de la population, valeurs dominantes, structures familiales) voire environnemental (par ex. climat $)^{19}$. Sans une telle approche qui permet de comprendre le fonctionnement, les tenants et les aboutissants des systèmes de santé, tout apprentissage tiré des comparaisons internationales semble illusoire.

On pourrait multiplier à l'envie les points qui relèguent les comparaisons internationales des systèmes de santé au rang de «tourisme intelligent $»^{20}$ mais ce qui est remarquable, comme d'autres l'ont noté, c'est qu'en matière de politique de santé « il y a un déséquilibre extraordinaire entre l'ampleur et la rapidité des flux d'information et la capacité de tirer des enseignements utiles $\gg{ }^{21}$. Dans ces conditions pourquoi ces comparaisons font-elles tant florès auprès des décideurs publics ? L'explication réside dans la convergence des propriétés scientifiques et politiques de la comparaison et celles du domaine de la santé, à la fois hautement technique et politique.

\section{Le mirage persistant de l' « evidence-based policy »}

Constatant l'obsolescence des connaissances médicales des médecins et la routinisation de leurs pratiques, au début des années 1990 des chercheurs formulèrent un plaidoyer en faveur d'une evidence-based medicine, c'est-à dire un exercice de la médecine fondé systématiquement sur des connaissances scientifiquement établies. Initialement centré sur la pratique, ce mouvement a rapidement atteint la décision publique sous le nom d'evidence-based policy ${ }^{22}$. Une décennie plus tard cette approche s'était diffusée à l'ensemble des domaines d'action publique, en commençant par celui de la santé. Fondamentalement, le succès de cette démarche dans le champ des politiques de santé tient au fait que les politiques 
sont associées à la pratique. Tout comme les pratiques, les politiques de santé pourraient ne plus être fondées sur des a priori mais au contraire être evidence-based. Si les soins peuvent être fondés sur des preuves, quoi de plus logique finalement que les politiques destinées à garantir la santé puissent l'être également ? Et quel meilleur moyen que la comparaison pour faire la preuve qu'une politique est pertinente? La comparaison est en effet généralement considérée dans les sciences sociales - qui vont appuyer la décision politique - comme le substitut de l'expérimentation dans les sciences naturelles. Rappelons que selon Durkheim, seule la comparaison permet d'administrer la preuve : «nous n'avons qu'un moyen de démontrer qu'un phénomène est cause d'un autre, c'est de comparer les cas où ils sont simultanément présents ou absents $»{ }^{23}$. On trouve dans cette congruence entre les propriétés du domaine de la santé et celles de la comparaison une première explication de la prospérité des comparaisons internationales des systèmes de santé.

Mais si l'on s'en tient à ce fondement, ce succès n'est peut-être pas si mérité. Il repose en fait sur une vision naïve des politiques publiques et tout particulièrement des politiques de santé où celles-ci pourraient ne pas être orientées par des valeurs, où les preuves peuvent être décontextualisées et où la fabrique des politiques se résumerait à un exercice scientifique rationaliste $^{24}$. Or, cette dépolitisation des politiques de santé est largement chimérique, elle oublie que les politiques de santé se fondent sur des choix subjectifs qui sont éminemment dépendants des contextes socio-politiques dans lesquels ils sont effectués. Qualité, équité et efficacité sont ainsi les critères principaux qui permettent d'identifier les bons systèmes de santé. Mais il s'agit aussi d'objectifs qui entrent en concurrence et dont la hiérarchisation s'établit en fonction des valeurs. La réponse à quel est le meilleur système - ou même seulement le plus adapté - dépendra donc toujours de celui à qui on pose la question (le patient, le médecin, le directeur d'hôpital, le contribuable, etc.) et de l'environnement sociopolitique dans lequel il évolue.

Un autre obstacle majeur au développement d'evidence-based policies fondées sur la comparaison réside dans la transférabilité des dispositifs $^{25}$. Les travaux comparatifs préconisant le transfert de solutions sur le fondement de comparaisons se concentrent généralement sur le système et ses outputs. Ils se demandent si les dispositifs adoptés à un endroit donné ont livré des résultats satisfaisants et, donc, méritent d'être importés ou exportés. Les inputs sont quant à eux négligés. En d'autres termes, ces travaux ne s'intéressent guère aux modalités de formulation et d'adoption des politiques publiques. Or, le succès d'un dispositif ne garantit en rien qu'il soit une solution envisageable ou réalisable ailleurs. Ces solutions vont être traduites en fonction des rapports de force politique, syndicaux ou encore des habitudes des acteurs. Par conséquent, dans certains contextes elles seront purement et simplement rejetées par l'organisme sur lequel on tente de les greffer. Ce fut le cas par exemple au début des années 1990 en Nouvelle-Zélande lors de l'introduction de frais hospitaliers à la charge du patient inspirés du modèle américain ${ }^{26}$. Dans d'autre cas ces solutions pourront se révéler contre-productives ${ }^{27}$. Au fond, puisque cette démarche suppose des acteurs agissant selon une absolue rationalité et en quête de la meilleure 
politique pour résoudre un problème, elle élude à la fois la dimension politique (i.e. les dispositifs sont liés à des valeurs), la dimension stratégique (i.e. les politiques sont l'objet de rapport de force) et la dimension cognitive de l'action publique (i.e. les représentations des acteurs et celles de la société encadrent la formulation des politiques publiques en permettant certaines options et en en éliminant d'autres). Enfin, les limites méthodologiques usuelles des comparaisons internationales évoquées plus haut sont autant d'éléments qui compliquent encore l'adoption d'evidence-based policies fondées sur des comparaisons.

Partant, les comparaisons internationales des systèmes de santé semblent conforter la dénonciation cinglante faite par Schultheis de l'exercice comparatif " se servant fréquemment de constructions de caractère scientifique » et qui « hérite d'une crédibilité sociale toute faite, ce qui explique entre autre son omniprésence dans les débats politiques où il sert d'ersatz et de " cache-misère »du raisonnement proprement dit ${ }^{28}$. Mais la paresse ou la croyance aveugle en l'existence d'une science du social seraient des explications simplistes du succès de la comparaison dans l'action publique, tout particulièrement dans le domaine de la santé. La rencontre des propriétés du domaine de la santé avec les propriétés de l'instrument comparaison conforte ce dernier pour une seconde raison : il est une ressource stratégique majeure.

\section{La comparaison comme arme politique}

La comparaison comme outil d'amélioration des politiques publiques repose sur deux principes : les décideurs choisiront le dispositif le plus per- tinent et les opposants potentiels l'accepteront parce qu'ils seront convaincus par la démonstration que c'est le meilleur choix possible. Pour le dire autrement, la comparaison est censée dépolitiser et rationaliser l'action publique en alimentant un double apprentissage : l'apprentissage des gouvernants et celui des opposants. Or, la comparaison impose souvent une solution plutôt qu'elle ne convainc de sa pertinence. Dans les domaines, tels que celui de l'organisation des systèmes de santé, « où les faits sont contestés, les valeurs sont complexes, et les différences partisanes sont nettes $»^{29}$, la comparaison tend particulièrement à jouer ce rôle de levier politique parce qu'elle contribue à la justification politique. Cet exercice, fondamental pour les décideurs politiques, passe effectivement par la réduction de la complexité des problèmes publics et la proposition de réponses logiques ${ }^{30}$. L'invocation de l'étranger permet l'un et l'autre à plusieurs titres.

D'abord, lorsqu'elle est mobilisée dans le champ politique, la comparaison simplifie le réel en fonctionnant par raccourci. Elle raconte une histoire qui commence par un problème et finit par une solution mais qui passe sur les rebondissements et ce qui se passe après le générique : on se contente d'affirmer que « l'exemple de la Suède/des ÉtatsUnis/de l'Allemagne/etc., confronté au même problème, montre que ce système fonctionne (ou pas) ». Elle fonctionne également par analogie : « si cela a marché (ou pas) en Suède/aux ÉtatsUnis/en Allemagne/etc., alors cela peut marcher (ou pas) ici ». Non seulement cette rhétorique simplifie le réel mais, dans la mesure où la comparaison repose sur une démarche scientifique - du moins s'en réclame, ne serait-ce qu'implicitement - elle impose une vérité, elle garantit la 
« certitude » écrivait Gusfield ${ }^{31}$. Comme il ne peut exister qu'une vérité scientifique, la comparaison interdit alors le débat.

La sémantique de la comparaison faisant d'elle un instrument tactique passe également par le langage déployé. La comparaison s'exprime fréquemment à travers des outils tels que des tableaux, des pourcentages, des courbes, des cartes et autres schémas (histogrammes, barres, bulles, etc. $)^{32}$. Ce langage de la comparaison possède une première particularité ; il s'agit d'un langage de rationalité scientifique. Ce langage où le chiffre est roi est celui de la neutralité et de la vérité. Ces outils d'apparat de la scientificité transforment des données et des résultats discutables en faits impartialement démontrés, donc incontestables ${ }^{33}$. Une deuxième particularité de ce langage réside dans sa grande aptitude communicative ${ }^{34}$. D'une part, le langage de la comparaison est adapté aux formats des medias de masse ; il permet de diffuser un message avec concision : un titre annonçant un résultat, un schéma pour l'illustrer et quelques phrases de commentaire. D'autre part, il rend le message universellement intelligible. En effet, en observant qui figure en haut ou en bas d'un classement, qui figure en vert ou en rouge sur une carte, qui voit sa courbe monter ou descendre ou encore qui a la barre la plus grande ou la plus petite dans un histogramme, chacun est en mesure de comprendre qui sont les bons et les mauvais élèves en comparaison. La portée stratégique de la comparaison est d'autant plus grande que questionner ses résultats n'est pas donné à tout le monde et une contre-analyse a peu de chance d'être menée par les medias ou même seulement d'être diffusée - devant nécessairement argumenter elle perdrait l'avantage de la simplicité et de la concision. Le classement des systèmes de santé par l'OMS en 2000 a fourni une illustration magistrale de ces logiques. Cette comparaison, présentée par le directeur général de l'OMS comme un instrument d'apprentissage evidence-based ${ }^{35}$, a trouvé un formidable écho dans les medias du monde entier. En revanche, le feu de critiques qu'il a subi, notamment dans la littérature académique, n'a lui guère été médiatisé ${ }^{36}$.

Plus largement, la comparaison peut produire une pression politique qui s'oppose par définition à l'apprentissage. Cette pression politique fonctionne selon les mêmes ressorts que la pression sociale, dont elle peut d'ailleurs être une conséquence. Il paraît ici important de rappeler que cette expression parfois utilisée hâtivement renvoie à des phénomènes démontrés par la psychologie sociale depuis les années 1950, dont le conformisme. Pour faire bref, Solomon Asch a démontré expérimentalement qu'un sujet tend à adopter l'opinion majoritairement exprimée par un groupe bien qu'à l'origine il soit convaincu qu'elle n'est pas pertinente : l'isolement produit le doute et la comparaison spontanée à la position des autres conduit au conformisme ${ }^{37}$. Mais on peut considérer que la probabilité de réaction conformiste redouble en politique. En effet, dans l'expérience de Asch, le sujet est conduit à se comporter ainsi alors même qu'aucun argument n'est déployé pour le convaincre et que son attitude n'engendre aucune conséquence : ni récompense, ni punition. Or, maintenir un choix hétérodoxe de politique publique alors que d'autres options sont soutenues par des argumentations comparatives - exposées auprès du public à l'aide de ces techniques si 
particulières - c'est prendre un risque majeur de sanction électorale. Tout particulièrement dans le domaine politiquement sensible de la santé.

Dès lors, la comparaison apparaît comme un outil clef de légitimation/délégitimation des choix politiques. La motivation de la comparaison peut d'ailleurs plus relever de la légitimation/ délégitimation que de l'apprentissage. Alors que la comparaison est censée permettre un apprentissage fondé sur les faits, l'élève connaît souvent la leçon avant de l'avoir apprise. En d'autres termes, les décideurs ont parfois forgé leur avis avant de recourir à la comparaison, ils n'invoquent l'étranger que pour légitimer des choix déjà effectués ou leur donner plus de substance ${ }^{38}$. Le Royaume-Uni de la fin des années 1980 illustre cette tendance des décideurs à tirer des leçons de manière sélective de l'expérience étrangère. Le gouvernement conservateur cherchant à mettre en œuvre des solutions libérales s'est tourné vers des comparaisons avec le modèle américain, qu'il savait correspondre à cette orientation, pour légitimer ses choix ${ }^{39}$. La comparaison ne serait alors qu'un outil supplémentaire non pas d'evidencebased policy mais de policy-based evidence $e^{40}$.

\section{Conclusion}

Au total, la comparaison internationale des systèmes de santé est loin de se réduire à un outil neutre d'action publique. Même si elle constitue plutôt un instrument intermédiaire ${ }^{41}$, la comparaison revêt la plupart des traits des « instruments d'action publique $»^{42}$. De fait, derrière un substrat technique qui laisse à penser qu'elle permet de gouverner « comme par raison $»^{43}$, la comparaison n'incarne pas une rationalité technique pure mais est porteuse de représentations du problème, de modes de régulation, de valeurs et d'intérêts. La frénésie comparative contribue à représenter les systèmes de santé comme étant strictement inscrits dans le cadre national. Elle propage l'image de systèmes de santé partout menacés et appelant des réformes urgentes. Elle laisse également penser que les systèmes de santé peuvent être gouvernés par les preuves. Par conséquent, il ne saurait y avoir de débats en valeurs autour de leurs réformes. Et cela d'autant plus que le langage à travers lequel la comparaison se déploie crée des effets de vérité qui légitiment ou délégitiment des choix de politique. La comparaison profite alors à ceux qui ont les moyens d'y recourir - notamment ceux qui disposent des données brutes ou qui peuvent financer cet exercice coûteux. Au-delà de sa fonction apparente de dépolitisation des enjeux, la comparaison est bien un instrument éminemment politique. D'une part, elle préformate les problèmes et les solutions. D'autre part, elle favorise les acteurs qui disposent des ressources adéquates. Au final, c'est donc peut-être plus parce qu'elle est un instrument de pouvoir que parce que qu'elle serait un outil d'apprentissage des bonnes pratiques que la comparaison internationale rencontre un tel succès auprès des acteurs publics. 
$\mathrm{N} \cdot \mathrm{O} \cdot \mathrm{T} \cdot \mathrm{E} \cdot \mathrm{S}$

1. Communiqué de presse du HCSP sur le rapport $L a$ santé en France et en Europe : contraste et convergence, Paris, 23 mai 2012.

2. O. Ihl (dir.), Les « sciences » de l'action publique, Grenoble, Presses Universitaires de Grenoble, 2005.

3. A. Smith, «L'analyse comparée des politiques publiques : une démarche pour dépasser le tourisme intelligent? », Revue internationale de politique comparée, 7 (1), 2000, p. 15.

4. P. Lascoumes, P. Le Galès (dir.), Gouverner par les instruments, Paris, Presses de Sciences Po, 2004.

5. T. Marmor, "Varieties of comparative analysis in the world of medical care policy », Journal of Comparative Policy Analysis: Research and Practice, 12 (1-2), 2010, pp. 5-10.

6. S'ils partagent de nombreux traits, comparaison et benchmarking ne se confondent pas totalement puisque ce dernier consiste à comparer par rapport à un étalon. I. Bruno, E. Didier, Benchmarking. L'Etat sous pression statistique, Paris, La Découverte, 2013

7. R. Blank, V. Burau, Comparative Health Policy, 3rd edition, Basingstoke, Palgrave Macmillan, 2010, p. 6. 8. Comme le suggèrent de Verdalle $\&$ al., cette atténuation des frontières entre sphère politique et académique, entre approche normative et scientifique de la comparaison, n'est pas propre au domaine de la santé. De Verdalle L., Vigour C., Le Bianic T., «S'inscrire dans une démarche comparative. Enjeux et controverses ", Terrains et travaux, 21 (2), 2012, pp. 5-21

9. A. Schneider, H. Ingram, « Systematically pinching ideas : a comparative approach to policy design », Journal of Public Policy, 8 (1), 1988, pp. 61-80.

10. R. Blank, V. Burau, 2010, op. cit., p. 7.

11. Sur ces critiques, voir G. Sartori, « Comparing and Miscomparing », Journal of Theoretical Politics, 3 (3),
1991, pp. 243-257 et P. Hassenteufel, « De la comparaison internationale à la comparaison transnationale. Les déplacements de la construction d'objets comparatifs en matière de politiques publiques ", Revue française de science politique, 55 (1), 2005, pp. 113-132.

12. OECD, Panorama de la santé 2009: Les indicateurs de l'OCDE, OECD Publishing, 2009.

13. T. Marmor, R. Freeman, K. Okma, « Comparative Perspectives and Policy Learning in the World of Health Care », Journal of Comparative Policy Analysis, 7 (4), 2005, pp. 331-348.

14. K. Okma K. \& al., « Six countries, Six health reform models ? Health care reform in Chile, Israel, Singapore, Switzerland, Taiwan and the Netherlands », Journal of Comparative Policy Analysis, 12 (1-2), 2010, pp. 75-113.

15. En France, la multiplication de dispositifs de déremboursement offre un exemple de ces pseudoréformes.

16. P. Hassenteufel, « Deux ou trois choses que je sais d'elle. Remarques à propos d'expériences de comparaisons européennes », in CURAPP, Les méthodes au concret, Paris, PUF, 2000, p. 108.

17. L'interprétation de l'évolution des systèmes de santé en termes de convergence globale est une autre illustration de cette logique. La convergence est souvent postulée, mal définie et sur-interprétée. Comme le relèvent R. Blank et V. Burau, en laissant penser que la convergence est inéluctable, les comparaisons internationales risquent de créer une prophétie autoréalisatrice qui pourrait conduire les décideurs à se laisser dicter des dispositifs inadaptés à leur contexte, R. Blank, V. Burau, 2010, op. cit., p.10

18. Pour des réflexions sur l'importance de ces dimensions dans la démarche comparative voir P. Hassenteufel, 2005, op.cit. ; C. Dupuy, J. Pollard, « La comparaison des politiques publiques infranationales : méthodes et pratiques », Revue internationale de poli- 
tique comparée, 19 (2), 2012, pp. 7-14.

19. R. Blank, V. Burau, 2010, op. cit.

20. A. Smith, 2000, op. cit.

21. T. Marmor, R. Freeman, K. Okma, 2005, op.cit., p. 331 .

22. C. Laurent \& al., « Pourquoi s'intéresser à la notion d'evidence-based policy? », Revue Tiers Monde, 200, 2009, pp. 853-873

23. E. Durkheim, Les règles de la méthode sociologique (1894), 10 éd., PUF, 1999, p. 124.

24. J. Russell, T. Greenhalgh, E. Byrne, J. McDonnell, « Recognizing rhetoric in health care policy analysis », Journal of Health Services Research \& Policy, 13 (1), 2008, pp. 40-46.

25. R. Klein, « Learning from others: Shall the last be the first? », Journal of Health Politics, Policy and Law, 22 (5), 1997, pp. 1267-1278.

26. R. Blank, V. Burau, 2010, op. cit., p. 249.

27. T. Marmor, R. Freeman, K. Okma, 2005, op.cit. Plus généralement, sur l'échec du transfert de politiques publiques, voir D. Dolowitz, D. March, « Learning from abroad: the role of policy transfer in contemporary policy making », Governance, 13 (1), 2000, pp. 5-24.

28. F. Schultheis, «Comme par raison - comparaison n'est pas toujours raison. Pour une critique sociologique de l'usage social de la comparaison interculturelle », Droit et société, n 11-12, 1989, p. 223.

29. D. B. Robertson, «Political conflict and lessondrawing », Journal of Public Policy, 11(1), 1991, p. 55. 30. Ibid, p. 57.

31. J. R. Gusfield, The culture of public problems: drinking-driving and the symbolic order, Chicago, The University of Chicago Press, 1981.

32. S. Guigner, « L'influence de l'Union européenne sur les politiques et pratiques de santé publique : européanisation verticale et horizontale », Sciences Sociales et Santé, 29 (1), 2011, pp. 81-106.
33. A. Desrosières, L'Argument statistique. Gouverner par les nombres (tome II), Paris, Presses de l'École des Mines, 2008.

34. Voir l'excellente illustration qu'en donne I. Bruno à partir de la comparaison européenne des investissements dans la R\&D ; I. Bruno, «Y a-t-il un pilote dans l'Union? Tableaux de bord, indicateurs, cibles chiffrées : les balises de la décision ", Politix, 82, 2008, pp. 95-118.

35. A. Williams, « Science or marketing at WHO? A commentary on 'World Health 2000'», Health Economics, 10, 2001, pp. 93-100 ; V. Navarro, « Assessment of the World health report 2000 », The Lancet, 356, 2000, pp. 1598-1601

36. Notons que si pour l'OMS ce rapport a visiblement servi d'outil marketing, les acteurs politiques l'ont eux utilisé comme arme politique. Pour ne mentionner que lui, le gouvernement espagnol a ainsi mis en avant le bon classement de l'Espagne dans cette comparaison pour défendre le statu quo alors qu'il était confronté à cette époque à des manifestations d'ampleur contre les délais de soins, V. Navarro, 2000, op.cit.

37. S. Asch, «Opinions and social pressure », Scientific American, 193 (5), 1955, pp. 31-35.

38. C. Bennett, «How states utilize foreign evidence », Journal of Public Policy, 11 (1), 1991, pp. 31-54.

39. R. Blank, V. Burau, 2010, op. cit.

40. Pour une autre illustration voir E. Chelle, Gouverner les pauvres. Politiques sociales et administration du mérite, Rennes, Presses Universitaires de Rennes, 2012, p. 247.

41. En effet, la comparaison contribue à définir l'action publique plus qu'elle ne la matérialise (comme le font par exemple les instruments réglementaires, budgétaires ou encore contractuels).

42. P. Lascoumes, P. Le Galès (dir.), 2004, op. cit.

43. F. Schultheis, 1989, op.cit., p. 223. 


\title{
$R \cdot E ́ \cdot S \cdot U \cdot M \cdot E ́$
}

Le détour par la comparaison internationale est devenu incontournable pour qui entend gouverner les systèmes de santé. Ces comparaisons favoriseraient l'apprentissage et permettraient de rationnaliser l'action publique. Mais leurs nombreuses failles font douter de leur rôle réel. La comparaison internationale s'apparente en fait à un « instrument d'action publique » : derrière son substrat technique et sa neutralité apparente, elle porte des représentations sociales, des valeurs, des intérêts et des rapports de force. Sous couvert de dépolitisation de la décision, la comparaison internationale est en réalité une puissante arme politique.

\begin{abstract}
International comparisons have become unavoidable for anyone who wants to govern health systems. Comparisons would foster learning processes and rationalize public policies. But the many flaws of these comparisons cast doubt on their actual role. Indeed, international comparisons are similar to «policy instruments»: behind their technical substrate and their apparent neutrality they carry social representations, values, interests and power relations. Under the guise of depoliticising the decisions international comparisons are actually powerful political weapons.
\end{abstract}


